\title{
Tools for studying composite models
}

\author{
Luca Panizzi ${ }^{\mathrm{a}}$ \\ Department of Physics and Astronomy, Uppsala University, Box 516, 75120 Uppsala, Sweden
}

Received 31 October 2020 / Accepted 7 July 2021 / Published online 11 August 2021 (C) The Author(s) 2021

\begin{abstract}
This contribution provides an overview of available numerical tools for performing phenomenological studies related to the new physics predicted by composite scenarios. The overview focuses on modelindependent constructions and aims at identifying the differences and connections between the implementations of numerical models, mostly focusing on the simplified-model approach. Attention is given to which kind of studies can be performed with such tools, with emphasis to the Higgs sector, new vector-like quarks and new bosonic states. A discussion of strategies for the analysis non-minimal simplified scenarios is also provided.
\end{abstract}

\section{Introduction}

The diversity of theoretical scenarios for realising a compositeness theory involves the prediction of an ample spectrum of new objects to be searched for at colliders, both present and future. The potential for discovering such new particles depends on their nature and on which kind of collider is considered.

The LHC will be starting the Run 3 phase in $2022,{ }^{1}$ which is meant to reach the nominal integrated luminosity of $300 \mathrm{fb}^{-1}$ with a center of mass energy of 14 $\mathrm{TeV}$. The most recent phenomenological studies are thus focused on exploiting data from the previous runs to pose constraints on theoretical models and on trying to predict the exclusion and discovery reaches of new searches during Run 3 or beyond. Projections are often given for the future high-luminosity phase of the LHC, aimed at increasing the luminosity up to $3 \mathrm{ab}^{-1} .^{2}$ Future colliders can be either hadronic or leptonic ${ }^{3}$ and a vast literature is being produced to identify scenarios

\footnotetext{
a e-mail: luca.panizzi@physics.uu.se (corresponding $\underset{1}{\text { author }}$ )

1 See https://home.cern/news/news/accelerators/ ls2-report-new-schedule.

2 More details can be found in [1] and at https://project-hl-lhc-industry.web.cern.ch/content/ project-schedule.

3 Detailed information and references can be found in [2-6] or at https://fcc.web.cern.ch/Pages/default.aspx and https://clic.cern/ (for colliders at CERN), in $[7$, 8] or at http://cepc.ihep.ac.cn/ (Circular ElectronPositron Collider in China), in $[9,10]$ or at http://ww2. linearcollider.org/ILC (for the International Linear Collider in Japan) or in [11] or at https://cerncourier.com/a/ sketching-out-a-muon-collider/ (for muon colliders).
}

which would make the future experiments sensitive to specific new physics.

A synergy between theory and experiment is, therefore, crucial to optimise the potential of new analyses by focusing on the most promising signatures of new physics predicted by composite models. To achieve that, the design of numerical models which contain the main features of scenarios of compositeness is a key step. The transposition from theory to numerical code is however not always straightforward given the potential complexity of theoretical models and their peculiar features, such as mass spectra, spins of new particles and their interactions between themselves and with the standard model (SM).

Testing specific theoretical benchmarks in the absence of any robust clue of new physics (except some anomalies) is a risky strategy, given the large number of possible theories which go beyond the SM, not only including compositeness realisations. A bottom-up approach based on the identification and study of signatures which can arise from classes of models has been thus a guiding principle in recent years. The development of models based on an effective field theory (EFT) description of low energy physics, and of simplified models, where the SM is minimally extended only with the lightest states of new physics which can be directly produced at colliders, represents a powerful and modelindependent strategy to study a large range of scenarios predicting particles with analogous properties. The possibility to allow for simulations at higher orders in the loop expansion with such models has further increased the accuracy with which results can be produced.

The interaction between the theoretical and experimental communities benefits also from the possibility to recast experimental searches and re-interpret their results in terms of theoretical scenarios which can 
be different, in principle, from those targeted by such searches. This allows to effectively exploit a large number of experimental results to test a wide range of new signals. The development of recasting tools to reconstruct experimental signal regions has greatly improved the potential for constraining the parameter spaces of theoretical models.

Finally, the development of specific simulation strategies and model-independent parametrisations for the analysis of signatures from non-minimal constructions can allow the reinterpretation of results from recasts in terms of a wide range of scenarios with different levels of complexity, and greatly help the design of new searches.

This contribution will treat the aforementioned aspects. After describing in Sect. 2 which kind of particles are mostly expected to provide signals compatible with composite models, an overview of numerical models available in the literature is provided in Sect. 3, together with a list of the main tools for the recasting of experimental searches. Finally, a discussion of parametrisations suitable for model-independent analyses of nonminimal scenarios, containing states with large width and/or with exotic decays, is presented in Sect. 4.

\section{What to search for at colliders}

After the discovery of the Higgs boson, focus has been given in precisely measuring its properties. Therefore, the obvious starting point to explore the compositeness hypothesis is the analysis of Higgs signatures, and of their possible deviations from the predictions of the SM. The determination of its mass and spin, of its width and of the strength of its couplings can indeed set constraints on the kind of new physics possibly connected to the Higgs, and in particular, composite interpretations [12-20]. Precision studies of processes involving the Higgs are the subject of an intense phenomenological and experimental effort. ${ }^{4}$

Hadron colliders are, however, suitable for the exploration of signatures coming from the direct production of new heavy coloured objects. In the context of composite scenarios, the presence of new quarks of vectorlike nature (VLQs) is usually a key ingredient, as they are for example invoked to generate the mass of SM fermions through their mixing with heavier composite fermions in partial compositeness scenarios [21-23].

VLQs have been largely studied [24-31] and searched at the LHC Runs 1 and 2, both in the pair and single production channels. ${ }^{5}$ Experimental studies usually relied on simplified models where the VLQs are added

\footnotetext{
4 The web page of the working group is https://twiki.cern. ch/twiki/bin/view/LHCPhysics/LHCHXSWG\#Overview.

5 The full list of searches can be found at the ATLAS and CMS web pages, https://twiki.cern.ch/twiki/bin/ view/AtlasPublic/WebHome\#Physics_Analysis_Groups_ Summary and http://cms-results.web.cern.ch/cms-results/ public-results/publications/B2G/index.html.
}

to the SM and only interact with SM particles. In the vast majority of such analyses, the VLQs have the same charge of SM quarks and they usually mix with the third generation of SM quarks. Searches have also been made for VLQs with exotic charges, and a few older searches, at 7 and $8 \mathrm{TeV}$, considered the single production of VLQs mixing with light generations. These choices have been dictated by the need to reduce to the minimum the complexity of the signatures of VLQs, in the hope that they were light enough to appear soon in experimental data. The absence of hints of new physics, however, has pushed the limits on the masses for such simplified scenarios above the $\mathrm{TeV}$ (the exact values depending on the type of VLQ and on its decay channels), both for extreme cases in which BRs into specific decays is saturated to $100 \%$ and for consistent constructions where specific representations of VLQs (singlets, doublets or triplets) are added to the SM, fixing the relations between the BRs.

More recently, phenomenological studies have been focusing on less minimal models (not only inspired by compositeness) where the VLQs can decay to new scalars or vectors, either neutral or charged [32-40]. Other studies have dealt with the possible presence of multiple VLQs, discussing the interplay between representations, interference effects and the corresponding reinterpretation of bounds [41-44]. The main motivations for extending the minimal simplified implementations and allow VLQs to interact also with further new states or with other VLQs is that theoretical models are always more complex than just a minimal extension of the SM: for example, new spin-0 objects can be lighter than VLQs and contribute significantly to their decay channels, or be the exclusive decay possibility [35], and the presence of VLQs with the same charge in multiplets of larger symmetries can be modelled by studying the interplay between multiple representations of VLQs.

The obvious phenomenological advantage in this kind of analyses is that by reducing the probability of decay into SM bosons, the limits on the masses of the VLQs from experimental searches can be reinterpreted and potentially lowered, opening the possibility to explore the larger parameter space corresponding to the presence of more interactions or of more step in the decay chain. Nevertheless, new final states can be constrained by different kind of experimental analyses, not necessarily aiming at detecting VLQs. The recasting of a wider set of searches, including SM measurements, can, therefore, still pose stringent bounds on VLQs in nonminimal setups $[33,38,45]$. If, for example, a VLQ toppartner $T$ is allowed to decay to a new neutral scalar $S$, the bounds on its mass depend not only on the corresponding branching ratio $B R(T \rightarrow S t)$, but also on the mass of $S$ and on its decay channels: assuming $T$ decays only into $S t$ and conditions for the narrow-width approximation (NWA) apply for both $T$ and $S$ decays [46], then the bounds are reduced to $600-800 \mathrm{GeV}$ for $S$ decaying to $\gamma \gamma$ or $\gamma Z$ [38], while they can be around the $\mathrm{TeV}$ if $S$ decays to $b \bar{b}$ or $g g$ [45] or $t \bar{t}[36]$. 
New spin-0 objects (including coloured ones) can also be directly searched at the LHC in different channels, and in some cases, signatures from the production and decays of new scalars can be very effective for constraining composite scenarios [47-53]. For non-coloured scalars, production modes include loop-induced processes analogous to the Higgs ones, in which both SM and new quarks can propagate, and strong constraints can be posed depending on their decays [54]. Bounds on the masses of new scalars can be in the multi-TeV range, depending, however, on the decay constants of the pNGBs of the various models. New spin-1 states are also predicted and have been phenomenologically studied with limits found to be in the multi-TeV range for most scenarios $[51,55,56]$.

As far as future lepton colliders are concerned, the possibility to perform precision physics would allow to increase the sensitivity in measuring the properties of the Higgs boson and therefore testing the compositeness scale [57]. Lepton colliders could also increase the potential for discovering light scalars, including those with very light masses (below the Z-boson mass) [58], and vector-like leptons, also predicted in composite scenarios [59].

\section{Numerical models and recasting tools}

This section is dedicated to a description of the main software tools for the study of composite models. Focus is given to numerical implementations of models to be used in Monte Carlo simulators and to analysis tools which allow to test the results of simulations against databases of recast experimental data.

\subsection{Numerical models}

To reproduce new physics signals in simulations for phenomenological or experimental analyses, the key elements are the numerical models. Such models can represent simplified scenarios or specific theoretical setups. In this section, attention will be mostly given to simplified models available in the literature, reporting the key elements of the Lagrangians, and how such models are available as pieces of software which can be used in Monte Carlo (MC) simulators. The common and different aspects of various implementations are identified, so that a comparison between results interpreted in different parametrisation can be made easier. The format of the models depends on which MC simulator is used to generate the signal. Focus will be given to models in the UFO [60] format which can be generated in FeynRules [61,62] or SARAh [63,64], to be used in MG5_aMC[65], GoSam [66], Sherpa [67], Whizard $[68,69]$ and Herwig [70], or to models in CALCHeP[71] format, which can be found in the repositories of FEYNRULES or HEPMDB[72].

As intimated in the previous section, the presence of new coloured fermions of vector-like nature in a large class of composite models makes such object of pri- mary interest for the exploration of these scenarios. Simplified scenarios have, therefore, been developed to describe the signatures arising from VLQs. The addition of VLQs to the SM is constrained by the possible representations under which a VLQ can transform, assuming it can only couple with SM states and mix with SM quarks through the Higgs boson [24-26, 28, 29]. The SM can be minimally extended in this way by adding either of the following fields, listed according to their representations under $S U(2)_{L}$ :

- Singlets: $T$ or $B$, with hypercharges $Y=2 / 3$ and $Y=-1 / 3$ respectively, which can interact with lefthanded SM quarks;

- Doublets: $(X T),(T B)$ or $(B Y)$, with hypercharges $Y=7 / 6, Y=1 / 6$ and $Y=-5 / 6$ respectively, which can interact with right-handed SM quarks;

- Triplets: $(X T B)$ or $(T B Y)$, with hypercharges $Y=2 / 3$ and $Y=-1 / 3$ respectively, which can interact with left-handed SM quarks;

for a total of seven possible VLQ representation, including both top- and bottom-partners $T$ and $B$, and exotic states $X$ and $Y$ with charges $5 / 3$ and $-4 / 3$, respectively. The connection between the dominant chirality of the couplings and the representations of the VLQs $[24,28]$ allows to interpret phenomenological analyses in the context of different theoretical realisations. The possibility of determining the dominant chirality of VLQs through the kinematical properties of the final states arising from their decay provides thus an important piece of information for the characterisation of the new physics associated with a potential VLQ discovery [73-75].

Model-independent Lagrangians which describe the interactions of VLQs with SM quarks and bosons have been developed in different analyses. They are all equivalent upon a redefinition of the coupling coefficients, but it is important to identify the differences in the conventions used in the parametrisations of the couplings. Re-interpretation of results from experimental searches in terms of the parameters of specific theoretical models can be strongly affected by such differences, especially for single production processes, where the cross-section is directly proportional to powers of the EW couplings through which the VLQs interact with SM quarks and bosons. In the following one of such parametrisations is considered explicitly, and the others are simply summarised in Table 1, through which it is possible to map the parametrisations into each other.

The Lagrangian of a simplified model describing the SM with the addition of one VLQ, taken as reference for the purposes of this contribution, is the one of Ref. $[76]:^{6}$

\footnotetext{
${ }^{6}$ The model is available in the NLO section of the FEynRules website at http://feynrules.irmp.ucl.ac.be/ wiki/NLOModels.
} 


$$
\begin{aligned}
\mathcal{L}= & -\sum_{Q=T, B}\left[h \bar{Q}\left(\hat{\kappa}_{L}^{Q} P_{L}+\hat{\kappa}_{R}^{Q} P_{R}\right) q+\text { h.c. }\right] \\
& +\frac{g}{2 c_{W}} \sum_{Q=T, B}\left[\bar{Q} \not\left(\tilde{\kappa}_{L}^{Q} P_{L}+\tilde{\kappa}_{R}^{Q} P_{R}\right) q+\text { h.c. }\right] \\
& +\frac{g}{\sqrt{2}} \sum_{Q=X, T, B, Y}\left[\bar{Q} W\left(\kappa_{L}^{Q} P_{L}+\kappa_{R}^{Q} P_{R}\right) q+\text { h.c. }\right],
\end{aligned}
$$

where $g=e / s_{W}$ corresponds to the weak interaction coupling of the SM and $s_{W}\left(c_{W}\right)$ is the sine (cosine) of the Weinberg angle. The couplings in front of the projectors are free parameters in the numerical model, but in any consistent Lagrangian describing the VLQ sector, they are related to the mixing parameters between the VLQ and the SM quarks, and have to be computed separately by the user. The numerical implementation of this lagrangian allows for the possibility to include NLO QCD corrections in Monte Carlo simulations. A crucial point in such parametrisation is that the coupling to the Higgs boson does not depend explicitly on the quark masses. If this was not the case, the renormalisation of the coupling would be affected by the renormalisation of masses, spoiling the cancellation of UV divergences emerging from the NLO calculation. The detailed procedure for obtaining the NLO numerical model and how UV divergences are treated can be found in the NLOCT reference [77]. The determination of K-factors to correct the cross-sections for pair and single production processes is complemented by the possibility to extract corrections at differential level, which allow to describe the kinematics of final states with increased accuracy [76,78].7,8,9

Studies describing the interplay between VLQs and the Higgs boson have also relied on non-public implementation of new states in MC simulators, such as in $[19,82]$ where the top partners have been included in Herwig [83].

Models containing a simplified description of the bosonic sector of composite scenarios have also been developed. In [84], a simplified model inspired by the effective Lagrangian for $S O(5) / S O(4)$ composite Higgs theories and describing spin-1 resonances interacting with SM states is introduced. ${ }^{10}$ Even if the coupling parameters and the masses of the spin- 1 resonances are free in the model, a Mathematica calculator is also provided to translate the input parameter in the Callan Coleman Wess Zumino formalism $[85,86]$ to the masses and couplings in the mass eigenstate basis.

\footnotetext{
7 The code can be found at http://jaguilar.web.cern.ch/ jaguilar/protos/.

8 The model is available in the FEYNRULES website at at http://feynrules.irmp.ucl.ac.be/wiki/VLQ.

9 The model is available in the HEPMDB website at https://hepmdb.soton.ac.uk/hepmdb:0214.0153.

10 The model is available in UFO format for MG5_AMC on the HEPMDB web site at http://hepmdb.soton.ac.uk/ hepmdb:0214.0154.
}

Besides the simplified model approach, models which can describe the properties of the Higgs assuming that new physics is heavy enough to be parametrised through EFT frameworks are widely used in phenomenology. Different parametrisations of the effective interactions of the Higgs arising from a strong sector have been proposed, such as the Standard Model Effective Field Theory (SMEFT), the Higgs Effective Field Theory (HEFT) and many other variations. The treatment of the EFTs is vast and beyond the scopes of this contribution: a very detailed overview can be found for example in [87]. On the numeric side, different EFT realisations, in different bases [88-90], have been implemented in FEYNRULES ${ }^{11}$ and software codes are available to translate the operators between different bases, such as RosetTa [91] or WCXF [92].

The relation between EFT operators and specific composite realisations is of course of primary importance for the purpose of determining a connection between the parameters of the theory and the bounds which can be obtained in the EFT frameworks [93]. For example, in [94], the Wilson coefficients for the most relevant SMEFT operators are calculated under generic assumptions about the mass scale and couplings of the strong dynamics, while in [95], universal relations between the EFT coefficients of composite Higgs bosons interacting the electroweak gauge bosons are presented.

Models describing specific composite realisations have also been developed. While such models contain a full set of particles with specific interactions, they can be used for more complex studies involving the interplay of multiple states in the construction of topologies leading to the same final states, including non-trivial effects which cannot be described by minimal realisations.

In $[96,97]$, an effective Lagrangian describing the low energy features of the Minimal Composite Higgs Model (MCHM) with partial compositeness, named 4DCHM, is described and implemented numerically. ${ }^{12}$. This model allows to generate the spectrum and interactions starting from 13 parameters representing the strong coupling scale, the gauge couplings of the new sector, the mixing parameters between the elementary and composite sector and the Yukawa couplings of the composite sector.

In [98], a model based on the coset $S U(4) / S p(4)$ is introduced, containing bosonic states, both scalar and vector. ${ }^{13}$ The implementation of the model allows to build the spectrum and interactions of the new

\footnotetext{
11 These implementations can be found in the FEYNRuLES website et at: http://feynrules.irmp.ucl.ac.be/ wiki/HiggsCharacterisation http://feynrules.irmp.ucl.ac. be/wiki/SMEFT http://feynrules.irmp.ucl.ac.be/wiki/ BSMCharacterisation http://feynrules.irmp.ucl.ac.be/ wiki/SMEFTatNLO.

12 The model is available for CALCHEP in the HEPMDB website at https://hepmdb.soton.ac.uk/hepmdb: 1212.0120

13 The model is available in UFO format for MG5_AMC in the HEPMDB website at https://hepmdb.soton.ac.uk/ hepmdb:0416.0200.
} 
Table 1 Conversion table between the coupling factors for a $T$ singlet of different numerical models for VLQ studies

\begin{tabular}{l} 
References $T W q \quad T Z q \quad T H q$ \\
$\kappa_{L}^{T} \quad \tilde{\kappa}_{L}^{T} \quad \hat{\kappa_{L}^{T}}$ \\
\hline$[25,79] \quad-\mathrm{V}_{4 j} \quad-\mathrm{X}_{4 \beta} \quad \frac{g}{2 M_{W}} m_{T} \mathrm{X}_{4 \beta}$ \\
$m_{T}$ is the mass of the $T$ mass eigenstate $\left(m_{T} \simeq M\right.$ with $M$ the parameter of the VLQ mass term $\left.M \bar{T} T\right) . V$ is the \\
generalised CKM matrix in the presence of the VLQ and $X=V V^{\dagger}$. The Lagrangians have been implemented in the \\
software tool PROTOS [99] for the generation of pair and single production of the VLQs
\end{tabular}

$[28] \quad \kappa_{T} \sqrt{\frac{\zeta_{i} \xi_{W}^{T}}{\Gamma_{W}^{O}}} \kappa_{T} \sqrt{\frac{\zeta_{i} \xi_{Z}^{T}}{\Gamma_{Z}^{O}}} \kappa_{T} \sqrt{\frac{\zeta_{i} \xi_{H}^{T}}{\Gamma_{H}^{O}}} \frac{M}{v}$

$\Gamma_{i}^{0}$ are the total widths of SM bosons for vanishing SM quark masses; $M$ is the parameter of the VLQ mass term $M \bar{T} T$; $v$ is the Higgs vacuum expectation value; $\xi_{V}$ and $\zeta_{i}$ measure, respectively, the weight of the coupling between $T$ and the SM bosons $(V=W, Z, h)$, and the weight of the mixing between $T$ and quarks of different generations $(i=u, c, t)$, and they are related in such a way that $B R\left(T \rightarrow V q_{i}\right)=\xi_{V} \zeta_{i}$ [100]. This model contains a known issue related to the couplings between the VLQs and the Higgs boson: the chiralities of the couplings, associated to the $\zeta$ parameters, are inverted in the numerical implementation and therefore, to be used consistently, chiralities for the Higgs couplings should be switched

$[80,81] \quad \sqrt{2} c_{L}^{T W} \quad \frac{c_{L}^{T Z}}{c_{W}} \quad c_{L}^{T h}$

In the original implementation the weak interaction coupling is labelled as $g_{w}$ [101].

The reference Lagrangian is the one in Eq. (1) and for a singlet only the coupling corresponding to the interaction of $T$ with left-handed SM quarks are considered. Analogous relations hold for different VLQs and different representations, hence the $T$ singlet example is sufficient to describe the main differences

particles and translate them into the param_card of MG5_AMC through a dedicated PYTHON calculator which can be downloaded together with the model.

The list of models presented in this section is summarised in Table 2, specifying the main features of the model for a quick reference about their purpose and limitations.

\subsection{Recasting of experimental searches}

Simulations performed with numerical models such as those described in the previous section provide a description of the kinematics of final states associated to signals of new physics. The comparison of such signals with experimental data is the mandatory next step for the determination of constraints on the parameter space of new physics, both from model-independent and theoretically motivated perspectives. The recasting of experimental searches relies on the possibility to (approximately) reproduce the experimental selections and kinematical cuts with fast simulation software to re-apply them to different signals of new physics. The most straightforward way to perform a recast is to develop a custom code which filters the simulated events reconstructing the experimental signal regions. Such process can be sometimes problematic due to the approximated outputs of fast simulators and the difficulties to reproduce with sufficient accuracy the experimental data.

A valid and quick alternative for this comparison is given by recasting tools, which provide a user-friendly interface and a database of built-in recasting of experimental searches, already validated, which can be used to combine the most stringent bounds on the model under consideration. A further advantage of the availability of a database of searches is that it makes possible to test signals predicted by a specific scenario against measurements which are not necessarily tuned to probe the same kind of new physics.

The list of recasting tools for new physics scenarios is long. Here, attention is limited to general purpose recasting software, such as MADANALYsis $5[102,103]$ and CHECKMATE $[104,105]$ : the database of recast searches provided within these tools is large, but of course not all experimental searches are as effective in posing bounds on the parameters of composite models. While searches targeting VLQ decays into SM objects, or searches targeting bosonic resonances, can be more useful, searches which target final states with large missing transverse energy (usually designed to search for supersymmetric signals) are not generally effective, even if for example the search [106], recast in CHECKMATE and targeting a final state with missing transverse energy and multiple jets and leptons, has been used in [36] to provide strong bounds (around the TeV) on the mass of a $T$ VLQ decaying to $S t$ with $S \rightarrow t \bar{t}$.

Among the searches currently recast in MADANALYSIS 5 or CHECKMATE, only few are likely to be effective for constraining composite models. Limiting only to $13 \mathrm{TeV}$ recasts, the searches which can be used for composite models are a handful. The CHECKMATE database contains the recast of the search [107] targeting multi-top final states. In the MADANALYSIS 5 framework there are multiple choices. The CMS search [108] recast in [109], for example, targets signatures with pair production of resonances decaying into 
Table 2 Summary of public numerical models for composite studies

\begin{tabular}{|c|c|c|}
\hline$\underline{\text { References }}$ & Features & Software \\
\hline \multicolumn{3}{|c|}{ Effective field theories } \\
\hline$[88]$ & $\begin{array}{l}\text { HEFT: SM without Higgs }+1 \text { bosonic state } \\
(\text { spin- } 0, \text { spin- } 1 \text { or spin- } 2)\end{array}$ & Source in FEynRules \\
\hline [89] & SMEFT (Warsaw basis) & Source in FEynRules \\
\hline$[91]$ & SMEFT (mass basis) & $\begin{array}{l}\text { Source in FEynRules. The tool Rosetta } \\
\text { allows to convert to different bases. }\end{array}$ \\
\hline$[90]$ & SMEFT (Warsaw basis) & $\begin{array}{l}\text { Source in FEYNRULES. Suitable for } \\
\text { simulations at NLO QCD in MG5_AMC }\end{array}$ \\
\hline \multicolumn{3}{|c|}{ Simplified models } \\
\hline$[25,79]$ & VLQ simplified model & PROTOS \\
\hline$[28]$ & VLQ simplified model & Source in FEYnRulES \\
\hline$[80,81]$ & VLQ simplified model & Source in FEynRules \\
\hline$[76]$ & VLQ simplified model & $\begin{array}{l}\text { Source in FEYNRULES. Suitable for } \\
\text { simulations at NLO QCD in MG5_AMC }\end{array}$ \\
\hline$[84]$ & $\begin{array}{l}\text { Spin-1 resonances in } S O(5) / S O(4) \\
\text { composite Higgs }\end{array}$ & Source in FEynRules \\
\hline \multicolumn{3}{|c|}{ Specific models } \\
\hline$[96,97]$ & $\begin{array}{l}\text { 4D description of composite Higgs models } \\
\text { with partial compositeness. It contains } \\
\text { new spin-1 resonances and new fermions }\end{array}$ & CALCHEP format \\
\hline$[98]$ & $\begin{array}{l}S U(4) / S p(4) \text { composite Higgs. It contains } \\
\text { spin-0 and spin- } 1 \text { resonances }\end{array}$ & UFO format \\
\hline
\end{tabular}

If the source is in FEYNRULes, it is possible to build numerical models in different formats to use with different MC simulators

three jets and the results are interpreted in terms of constraints on fermionic colour octets, but the same search can also be used to pose constraints on VLQs decaying to exotic scalars. The search [110] recast in [111] targets a signal coming from the Higgs boson decaying into two light pseudo-scalars which subsequently decay into two bottoms and two muons: this search can be directly used to pose constraints to composite models (analogously to what has been done in [52] using the $8 \mathrm{TeV}$ CMS search [112]). Finally, searches looking at final states with four tops, as [113,114], recast respectively in $[115,116]$, can be effective for testing scenarios where new bosons decay to top pairs. A summary of the previously described searches is provided in Table 3 .

It must be stressed however that the possibility of implementing new searches is a powerful feature of both tools: if publicly available data from experimental searches allow to successfully validate a recast code, a user is allowed to reinterpret any experimental result to determine bounds of new physics and compute projections for higher luminosities.

Comparison of signals of new physics with SM measurements can also be very useful to constrain new physics scenarios, including signatures coming from composite models. The ConTuR framework [117], compares a new physics signal to searches aimed at performing precision measurement of SM (in contrast to searches aimed at discovering new particles) exploiting the RIVET library [118]. The results of such comparison can be indeed competitive with the recasting of searches dedicated to the discovery of new physics [45].

\section{Analysis strategies and representation of results: the case of VLQs with large width}

In many cases, the minimal implementations of simplified models are not sufficient to accurately describe the main aspects of new physics scenarios. More particles are often necessary, increasing the number of free parameters, as in the models described at the end of Sect. 2. Even just the presence of multiple interactions of the same particle are enough to increase the complexity of the problem and require the identification of specific benchmarks for the analysis.

Nevertheless, it is often possible to deconstruct the signal into elements which determine a specific kinematics for the objects in the final state, i.e. specific shapes of differential distributions. Such signal elements, when linearly combined with appropriate weights, can reconstruct different kind of more complex signals, characterised by different kinematics associated with the dominance of one or the other element depending on the weights. The advantage of this procedure is that the simulation of signals and interference contributions can be made modular, and the interplay between different topologies can be reconstructed a posteriori through the combination of objects from a databases of event files, each characterised only by the properties which influence the kinematical distributions (such as masses, spins and total widths of the particles).

As a practical examples, the case of VLQs with large width is considered.

Phenomenological studies and experimental searches of VLQs largely focus on scenarios where the total 
Table 3 A list of searches at $13 \mathrm{TeV}$ recast in MADANALYsis 5 or CHECKMATE which can be used to constrain signatures originating from composite scenarios

\begin{tabular}{|c|c|c|c|c|}
\hline References & $\begin{array}{l}\text { Luminosity } \\
\left(f b^{-1}\right)\end{array}$ & Final state & Target & Recasting tool \\
\hline ATLAS [107] & 3.2 & $\begin{array}{l}\text { One lepton, multiple } \\
\text { jets and MET }\end{array}$ & $\begin{array}{l}\text { Multi-tops with at } \\
\text { least one decaying } \\
\text { to Higgs }\end{array}$ & CHeckMATE \\
\hline CMS [108] & 35.9 & 6 jets & $\begin{array}{l}\text { Pair production of } \\
\text { gluinos with } \\
\text { R-parity violating } \\
\text { decays in } 3 \text { jets } \\
\text { each }\end{array}$ & MADANALYSis 5[109] \\
\hline CMS [110] & 35.9 & 2 b-jets and $2 \mu$ & $\begin{array}{l}\text { Higgs boson } \\
\text { decaying to two } \\
\text { light pseudoscalars }\end{array}$ & MADANALYSIS 5[111] \\
\hline CMS [113] & 35.9 & $\begin{array}{l}2 \text { same sign leptons } \\
\text { or } 3 \text { leptons }+ \\
\text { multiple jets and } \\
\text { b-jets }\end{array}$ & $\begin{array}{l}\text { SM production of } 4 \\
\text { top quarks }\end{array}$ & MADANALYSIS 5[115] \\
\hline CMS [114] & 137.1 & $\begin{array}{l}2 \text { same sign leptons } \\
\text { or } 3 \text { leptons }+ \\
\text { multiple jets and } \\
\text { b-jets }\end{array}$ & $\begin{array}{l}\text { SM production of } 4 \\
\text { top quarks }\end{array}$ & MADANALYSIS 5[116] \\
\hline
\end{tabular}

Any search can in principle be used, but those targeting final states with high jet, b-jet, top or lepton multiplicity and without strong cuts on missing transverse energy are preferable

width of VLQs is small with respect to their mass, the NWA. This allows to efficiently factorise the production and decays of the VLQs and to describe with excellent accuracy the kinematics of the final states. However, VLQs do not necessarily have to be narrow.

Partial widths are always proportional to the square of the corresponding interaction coupling, which means that regardless of the mass differences between the decaying particle and the decay products, the larger the couplings, the higher the possibility that the width of the particle becomes sizable with respect to its mass.

For the simplified models of Sect. 3, where the only interactions of VLQs are with SM particles, constraints have to be imposed on the size of the corresponding couplings, to comply with experimental observables coming from different source, ranging from collider, to flavour physics, to electroweak precision tests $[26,28,29,119$ 121]. Such constraints limit the size of VLQ couplings to values which are small enough for VLQs to be treated in the NWA [122]. Different assumptions have thus to be made to perform phenomenological analyses of scenarios with VLQ with large width. The presence of multiple VLQs with same charge, for example, can induce cancellations of contributions which can relax some of the constraints $[43,44]$ and allow for large couplings and in turn larger widths. A different possibility is represented by decays of VLQs into further new states besides the SM ones, implying that the total width of VLQs receives potentially sizable contributions from new decay channels.

Phenomenological studies have been performed for both pair [122,123] and single production [124] and experimental searches have explored the large width regime in VLQ single production [125-128]. While processes of pair production retain a certain degree of model-independency due to the fact that the crosssection is essentially driven by the mass of the VLQ, processes of single production crucially depend on the same EW couplings which also determine the VLQ width. Furthermore, the treatment of VLQs with large width has to take into account multiple effects: the contributions of events where the decay products have an invariant mass far from the resonant peak, the contribution of topologies where the VLQ does not propagate resonantly, which can provide sizable signal-signal interference contributions unlike in the NWA case, and finally the potentially enhanced interference between signal and SM background.

The kinematical properties of the final state depend on the mass and total width of the VLQ propagating in the signal and interference topologies. The corresponding cross-sections are proportional to different powers of the VLQ couplings, which can be factorised without affecting the shape of the kinematical distributions of the final states. Following the notation of Ref. [124] and further improving its original formulation, the signal for single production of a VLQ with large width can be parametrised as:

$$
\begin{aligned}
\sigma_{S}\left(C_{i}, C_{2}, M_{Q}, \Gamma_{Q}\right) & =C_{2}^{2} \sum_{i} C_{1 i}^{2} \hat{\sigma}_{S_{i}}\left(M_{Q}, \Gamma_{Q}\right), \\
\sigma_{S S}^{\mathrm{int}}\left(C_{i}, C_{2}, M_{Q}, \Gamma_{Q}\right) & =C_{2}^{2} \sum_{i \neq j} C_{1 i} C_{1 j} \hat{\sigma}_{S_{i} S_{j}}^{\mathrm{int}_{j}}\left(M_{Q}, \Gamma_{Q}\right),
\end{aligned}
$$




$$
\sigma_{S B}^{\mathrm{int}}\left(C_{i}, C_{2}, M_{Q}, \Gamma_{Q}, \chi_{Q}\right)=C_{2} \sum_{i} C_{1 i} \hat{\sigma}_{S_{i} B}^{\mathrm{int}}\left(M_{Q}, \Gamma_{Q}, \chi_{Q}\right) .
$$

The meaning of these expressions can be better understood by referring to an explicit example: the process $p p \rightarrow W^{+} \bar{b} t$ corresponding to the single production of a VLQ top-partner $T$ decaying into $W b$. This process is represented in Fig. 2 of Ref. [124] and reported here for clarity in Fig. 1.

- Equation 2 sums all the cross-sections corresponding to the squared amplitudes of the signal topologies, each proportional to the square of the coupling between the VLQ and the boson in the final state $\left(C_{2}\right)$ and the square of the coupling between the VLQ and the particles propagating in the topologies $\left(C_{1 i}\right)$. In the $p p \rightarrow W^{+} \bar{b} t$ example, and using the notation of Eq. 1 to label the couplings, this corresponds to the amplitude squared of topologies (a) and (b):

$$
\begin{aligned}
\sigma_{S}\left(\tilde{\kappa}_{\chi}^{T}, \kappa_{\chi}^{T}, M_{T}, \Gamma_{T}\right)= & \left(\kappa_{\chi}^{T}\right)^{2}\left(\left(\kappa_{\chi}^{T}\right)^{2} \hat{\sigma_{W}}\left(M_{T}, \Gamma_{T}\right)\right. \\
& \left.+\left(\tilde{\kappa}_{\chi}^{T}\right)^{2} \hat{\sigma_{Z}}\left(M_{T}, \Gamma_{T}\right)\right), \quad(5
\end{aligned}
$$

with $\chi=L, R$.

- Equation 3 sums the cross-sections corresponding to the interference between different signal topologies, proportional to the same factor $C_{2}^{2}$ and to the product of different $C_{1}$ couplings. In the $p p \rightarrow W^{+} \bar{b} t$ example, it corresponds to the interference between the (a) and (b) topologies:

$$
\begin{aligned}
& \sigma_{S S}\left(\tilde{\kappa}_{\chi}^{T}, \kappa_{\chi}^{T}, M_{T}, \Gamma_{T}\right) \\
& =\left(\kappa_{\chi}^{T}\right)^{2}\left(\kappa_{\chi}^{T} \tilde{\kappa}_{\chi}^{T} \hat{\sigma}_{W Z}\left(M_{T}, \Gamma_{T}\right)\right) .
\end{aligned}
$$

- Equation 4 sums the cross-sections associated to the interference between signal topologies and the SM background, each linearly proportional to the product of $C_{2}$ and the corresponding $C_{1}$ couplings. In the $p p \rightarrow W^{+} \bar{b} t$ example, it corresponds to the sum of the interferences between (a) and (c) and between (b) and (c):

$$
\begin{aligned}
\sigma_{S B}\left(\tilde{\kappa}_{\chi}^{T}, \kappa_{\chi}^{T}, M_{T}, \Gamma_{T}, \chi\right)= & \kappa_{\chi}^{T}\left(\kappa_{\chi}^{T} \hat{\sigma}_{W B}\left(M_{T}, \Gamma_{T}, \chi\right)\right. \\
& \left.+\tilde{\kappa}_{\chi}^{T} \hat{\sigma}_{Z B}\left(M_{T}, \Gamma_{T}, \chi\right)\right)
\end{aligned}
$$

The dominant chirality of the couplings $\chi_{Q}$ does not affect $\sigma_{S}$ and $\sigma_{S S}^{\text {int }}$ at the inclusive level, while the crosssections associated to the interference with the back- ground explicitly depends on $\chi_{Q}$ due to the fact that the background amplitudes are independent of $\chi_{Q}$.

Notice that as reported in the original formulation, the interference terms may introduce a gauge dependence in the definitions of $\sigma_{S}$ and $\sigma_{S S}^{\text {int }}$; however, unless the gauge sector of the SM is modified by other new physics, the quantities $\sigma_{S}+\sigma_{S S}^{\text {int }}$ and $\sigma_{S B}^{\text {int }}$ are gauge invariant. Therefore if all the individual $\hat{\sigma}$ of Eqs. 2 to 4 are computed for any $M_{Q}$ and $\Gamma_{Q}$ combination in a consistent choice of gauge, the signals arising from different scenarios characterised by the same mass and total width of the VLQ, but by different numerical values of its couplings (assuming that the sum of the corresponding partial width does not exceed $\Gamma_{Q}$ for consistency), can be computed by summing all the terms in the equations with the corresponding values of the couplings.

The same procedure can be applied at differential level, as the couplings only act as a rescaling of the individual shapes without deforming them. On the other hand, at the differential level, the chirality of the couplings has an impact on the shape of the distributions, and cannot, therefore, be factorised in the same way as the couplings. The role of the dominant chirality in affecting the shapes of final state distributions derives from the different polarizations of the SM states resulting from the decays of VLQs and it is known to affect the experimental bounds and future projections, especially if the VLQ decays to top quarks [73-75]. The sensitivity to the chirality-induced kinematical differences depends on the process and on the specific search: the chirality dependence can thus be encoded in the efficiency of selections and kinematical cuts by separately computing such efficiencies for dominant left-handed or right-handed couplings.

The whole discussion can be straightforwardly extended for scenarios involving multiple VLQs or involving different state propagating in the signal topologies, such as new scalars or vectors.

The representation of the results can then be provided in the $\{M, \Gamma / M\}$ plane for the reduced theoretical cross-sections $\hat{\sigma}[124]$ and for the efficiencies of selection and kinematical cuts [38]. Such representation allows for effective reinterpretations in scenarios where, for example, the partial widths associated to the couplings which enter the single production topologies do not sum up to the total width, which gets contributions from other channels.

Model-independent analyses for processes in nonminimal scenarios following the above procedure depend, however, on the possibility to isolate the signal and interference contributions and simulate them separately. This is possible in MG5_AMC with models which allow to specify the coupling orders of new couplings independently from the SM ones. A generic MG5_AMC syntax to isolate the contributions of Fig. 1 in the case of a $T$ VLQ with dominant left-handed couplings would read (the syntax is a template, not related to a specific numerical model):

$$
\text { generate } \mathrm{p} \mathrm{p}>\mathrm{b} \sim \mathrm{t} \mathrm{w}+\mathrm{CO} 1 \mathrm{CO} 2 \ldots \text {, }
$$




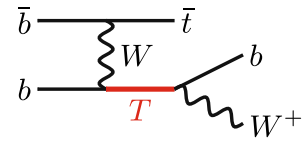

(a)

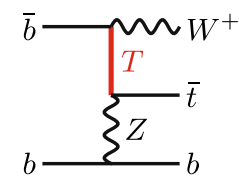

(b)
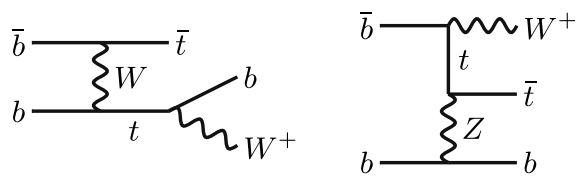

(c)

Fig. 1 Topologies for the single production process of a VLQ with charge $2 / 3, T$, in the five-flavour number scheme. The first two topologies represent the signal, where the $T$ interacts with both the $W$ and $Z$ bosons of the SM. The two right diagrams correspond to the SM background

where $\mathrm{CO} 1 \mathrm{CO} 2 \ldots$ represents a list of coupling orders assignment, corresponding to the couplings of the VLQ needed to reproduce the various topologies and interference terms. For the example of Fig. 1, such lists are represented by the following strings:

(string in common to all topologies) $\mathrm{TWR}=0 \mathrm{TZR}=0$ THR $=0$,

(a) $Q E D==1 \quad T W L==2 \quad T Z L=0 \quad T H L=0$,

(b) $Q E D==1 \quad T W L==1 \quad T Z L==1 \quad T H L=0$,

(c) $\mathrm{TWL}=0 \mathrm{TZL}=0 \mathrm{THL}=0$ or simply / $\mathrm{T}$ to completely exclude the propagation of the VLQ $T$ in SM diagrams,

(ab-interference) QED $==2 \mathrm{TWL}==3 \mathrm{TZL}==1 \mathrm{THL}=0$,

(ac-interference) $\mathrm{QED} 2==4 \mathrm{TWL}==2 \mathrm{TZL}=0 \mathrm{THL}=0$,

(bc-interference) $\mathrm{QED} 2==4 \mathrm{TWL}==1 \mathrm{TZL}==1 \mathrm{THL}=0$.

The possibility of selecting coupling orders is implemented in some of the models described above, as in the model of Refs. [80,81] or, after appropriate modifications, the models of $[28,76]$. The difference between the first model and the other two is that the first model already defines individual coupling orders for each coupling of the VLQs with different chiralities, while the other two define a generic coupling order for every interaction of the VLQs, which can be generalised for the purpose of signal deconstruction.

\section{Conclusions}

This contribution provided a brief overview of the tools for performing phenomenological analyses in the context of composite models, focusing on those inspired by a bottom-up approach. Such tools are meant to help studying processes which have a higher chance to lead to discoveries in the near future, involving the SM Higgs boson, new particles such as vector-like quarks, new bosonic states, and the interplay between them. The numerical models needed to the analysis of such processes in Monte Carlo simulators have been described, stressing their differences and peculiar features which can allow different types of studies. The role of recasting software for computing bounds on the parameter space of composite models has also been treated. Finally, specific model-independent analysis strategies have been presented, involving the deconstruction of signal and interference terms for the reinterpretation of results in a wide range of scenarios. The application of such strategies for vector-like quarks with large width, requiring specific features in the numerical models, has been discussed.
Acknowledgements This work is supported by the Knut and Alice Wallenberg foundation under the SHIFT project, grant KAW 2017.0100.

Funding Open access funding provided by Uppsala University.

Open Access This article is licensed under a Creative Commons Attribution 4.0 International License, which permits use, sharing, adaptation, distribution and reproduction in any medium or format, as long as you give appropriate credit to the original author(s) and the source, provide a link to the Creative Commons licence, and indicate if changes were made. The images or other third party material in this article are included in the article's Creative Commons licence, unless indicated otherwise in a credit line to the material. If material is not included in the article's Creative Commons licence and your intended use is not permitted by statutory regulation or exceeds the permitted use, you will need to obtain permission directly from the copyright holder. To view a copy of this licence, visit http://creativecomm ons.org/licenses/by/4.0/.

\section{References}

1. High-Luminosity Large Hadron Collider (HL-LHC): Preliminary Design Report (2015)

2. FCC collaboration, FCC Physics Opportunities: future circular collider conceptual design report volume 1. Eur. Phys. J. C 79, 474 (2019)

3. FCC collaboration, FCC-ee: The Lepton Collider: future circular collider conceptual design report volume 2. Eur. Phys. J. ST 228, 261 (2019)

4. FCC collaboration, FCC-hh: The Hadron collider: future circular collider conceptual design report volume 3. Eur. Phys. J. ST 228, 755 (2019)

5. FCC collaboration, HE-LHC: the high-energy large Hadron Collider: future circular collider conceptual design report volume 4. Eur. Phys. J. ST 228, 1109 (2019)

6. A multi-TeV linear collider based on CLIC technology: CLIC Conceptual Design Report (2012)

7. CEPC Study Group collaboration, CEPC Conceptual Design Report: Volume 1 - Accelerator (2018). arXiv:1809.00285

8. CEPC Study Group collaboration, CEPC Conceptual Design Report: volume 2 - Physics 85 Detector (2018). arXiv:1811.10545 
9. The International Linear Collider Technical Design Report - vol. 1: Executive Summary (2013). arXiv:1306.6327

10. The International Linear Collider Technical Design Report - Vol. 2: Physics (2013). arXiv:1306.6352

11. MAP, MICE collaboration, Muon colliders and neutrino factories. EPJ Web Conf. 95, 03019 (2015). arXiv: 1412.3487

12. T. Abe, R. Kitano, Phenomenology of partially composite standard model. Phys. Rev. D 88, 015019 (2013). arXiv:1305.2047

13. M. Montull, F. Riva, E. Salvioni, R. Torre, Higgs couplings in composite models. Phys. Rev. D 88, 095006 (2013). arXiv:1308.0559

14. A. Pich, I. Rosell, J. Sanz-Cillero, Oblique S and $\mathrm{T}$ Constraints on electroweak strongly-coupled models with a light Higgs. JHEP 01, 157 (2014). arXiv: 1310.3121

15. S. Dawson, E. Furlan, Yukawa Corrections to Higgs Production in Top Partner Models. Phys. Rev. D 89, 015012 (2014). arXiv:1310.7593

16. D. Ghosh, M. Salvarezza , F. Senia, Extending the analysis of electroweak precision constraints in composite Higgs models. Nucl. Phys. B 914, 346 (2017). arXiv:1511.08235

17. G. Cacciapaglia, H. Cai, A. Carvalho, A. Deandrea, T. Flacke, B. Fuks et al., Probing vector-like quark models with Higgs-boson pair production. JHEP 07, 005 (2017). arXiv:1703.10614

18. A. Belyaev, P. Schaefers, M. Thomas, Precise test of Higgs boson properties via triple Higgs boson production in vector boson fusion at future colliders. Phys. Rev. D 99, 015030 (2019). arXiv:1801.10157

19. A. Banfi, B.M. Dillon, W. Ketaiam , S. Kvedaraite, Composite Higgs at high transverse momentum. JHEP 01, 089 (2020). arXiv:1905.12747

20. G. Cacciapaglia, C. Pica , F. Sannino, Fundamental composite dynamics: a review. Phys. Rept. 877, 1 (2020). arXiv:2002.04914

21. D.B. Kaplan, Nucl. Phys. B 365, 259 (1991)

22. B. Gripaios, A. Pomarol, F. Riva, J. Serra, Beyond the minimal composite Higgs model. JHEP 04, 070 (2009). arXiv:0902.1483

23. G. Panico, A. Pomarol, Flavor hierarchies from dynamical scales. JHEP 07, 097 (2016). arXiv:1603.06609

24. F. del Aguila, M. Perez-Victoria, J. Santiago, Observable contributions of new exotic quarks to quark mixing. JHEP 09, 011 (2000). arXiv:hep-ph/0007316

25. J. Aguilar-Saavedra, Identifying top partners at LHC. JHEP 11, 030 (2009). arXiv:0907.3155

26. Y. Okada, L. Panizzi, LHC signatures of vector-like quarks. Adv. High Energy Phys. 2013, 364936 (2013). arXiv:1207.5607

27. G. Cacciapaglia, A. Deandrea, L. Panizzi, S. Perries, V. Sordini, Heavy vector-like quark with charge 5/3 at the LHC. JHEP 03, 004 (2013). arXiv:1211.4034

28. M. Buchkremer, G. Cacciapaglia, A. Deandrea, L. Panizzi, Model independent framework for searches of top partners. Nucl. Phys. B 876, 376 (2013). arXiv: 1305.4172

29. J. Aguilar-Saavedra, R. Benbrik, S. Heinemeyer, M. Pérez-Victoria, Handbook of vectorlike quarks: Mixing and single production. Phys. Rev. D 88, 094010 (2013). arXiv:1306.0572

30. O. Matsedonskyi, G. Panico, A. Wulzer, Top Partners Searches and Composite Higgs Models. JHEP 04, 003 (2016). arXiv:1512.04356

31. O. Panella, R. Leonardi, G. Pancheri, Y. Srivastava, M. Narain, U. Heintz, Production of exotic composite quarks at the LHC. Phys. Rev. D 96, 075034 (2017). arXiv:1703.06913

32. J. Serra, Beyond the Minimal Top Partner Decay. JHEP 09, 176 (2015). arXiv:1506.05110

33. J. Aguilar-Saavedra, D. López-Fogliani, C. Muñoz, Novel signatures for vector-like quarks. JHEP 06, 095 (2017). arXiv:1705.02526

34. M. Chala, Direct bounds on heavy toplike quarks with standard and exotic decays. Phys. Rev. D 96, 015028 (2017). arXiv:1705.03013

35. N. Bizot, G. Cacciapaglia , T. Flacke, Common exotic decays of top partners. JHEP 06, 065 (2018). arXiv:1803.00021

36. H. Han, L. Huang, T. Ma, J. Shu, T. M. Tait, Y. Wu, Six top messages of new physics at the LHC. JHEP $\mathbf{1 0}$, 008 (2019). arXiv:1812.11286

37. K.-P. Xie, G. Cacciapaglia , T. Flacke, Exotic decays of top partners with charge 5/3: bounds and opportunities. JHEP 10, 134 (2019). arXiv:1907.05894

38. R. Benbrik et al., Signatures of vector-like top partners decaying into new neutral scalar or pseudoscalar bosons. JHEP 05, 028 (2020). arXiv:1907.05929

39. G. Cacciapaglia, T. Flacke, M. Park, M. Zhang, Exotic decays of top partners: mind the search gap. Phys. Lett. B 798, 135015 (2019). arXiv:1908.07524

40. J. Aguilar-Saavedra, J. Alonso-González, L. Merlo , J. No, Exotic vectorlike quark phenomenology in the minimal linear $\sigma$ model. Phys. Rev. D 101, 035015 (2020). arXiv:1911.10202

41. D. Barducci, A. Belyaev, J. Blamey, S. Moretti, L. Panizzi, H. Prager, Towards model-independent approach to the analysis of interference effects in pair production of new heavy quarks. JHEP 07, 142 (2014). arXiv: 1311.3977

42. D. Barducci, A. Belyaev, M. Buchkremer, G. Cacciapaglia, A. Deandrea, S. De Curtis et al., Framework for model independent analyses of multiple extra quark scenarios. JHEP 12, 080 (2014). arXiv:1405.0737

43. G. Cacciapaglia, A. Deandrea, N. Gaur, D. Harada, Y. Okada, L. Panizzi, Interplay of vector-like top partner multiplets in a realistic mixing set-up. JHEP 09, 012 (2015). arXiv:1502.00370

44. G. Cacciapaglia, A. Deandrea, N. Gaur, D. Harada, Y. Okada , L. Panizzi, The LHC potential of Vector-like quark doublets. JHEP 11, 055 (2018). arXiv:1806.01024

45. G. Brooijmans et al., Les Houches 2019 Physics at TeV Colliders: New Physics Working Group Report (contribution 5). In 11th Les Houches Workshop on Physics at TeV Colliders: PhysTeV Les Houches, vol. 2 (2020). arXiv:2002.12220

46. D. Berdine, N. Kauer, D. Rainwater, Breakdown of the narrow width approximation for new physics. Phys. Rev. Lett. 99, 111601 (2007). arXiv:hep-ph/0703058

47. G. Cacciapaglia, H. Cai, A. Deandrea, T. Flacke, S.J. Lee , A. Parolini, Composite scalars at the LHC: the 
Higgs, the Sextet and the Octet. JHEP 11, 201 (2015). arXiv: 1507.02283

48. J. P. Araque, N.F. Castro, J. Santiago, Interpretation of vector-like quark searches: heavy gluons in composite Higgs models. JHEP 11, 120 (2015). arXiv:1507.05628

49. G. Ferretti, Gauge theories of Partial Compositeness: Scenarios for Run-II of the LHC. JHEP 06, 107 (2016). arXiv:1604.06467

50. D. Buarque Franzosi, F. Fabbri, S. Schumann, Constraining scalar resonances with top-quark pair production at the LHC. JHEP 03, 022 (2018). arXiv: 1711.00102

51. D. Liu, L.-T. Wang, K.-P. Xie, Prospects of searching for composite resonances at the LHC and beyond. JHEP 01, 157 (2019). arXiv:1810.08954

52. G. Cacciapaglia, G. Ferretti, T. Flacke, H. Serôdio, Light scalars in composite Higgs models. Front. Phys. 7, 22 (2019). arXiv:1902.06890

53. G. Cacciapaglia, A. Deandrea, T. Flacke, A. Iyer, Gluon-Photon signatures for color octet at the LHC (and beyond). JHEP 05, 027 (2020). arXiv:2002.01474

54. A. Belyaev, G. Cacciapaglia, H. Cai, G. Ferretti, T. Flacke, A. Parolini et al., Di-boson signatures as standard candles for partial compositeness. JHEP 01, 094 (2017). arXiv:1610.06591

55. A. Thamm, R. Torre, A. Wulzer, Future tests of Higgs compositeness: direct vs indirect. JHEP 07, 100 (2015). arXiv:1502.01701

56. D. Liu, L.-T. Wang , K.-P. Xie, Broad composite resonances and their signals at the LHC. Phys. Rev. D 100, 075021 (2019). arXiv:1901.01674

57. R. Contino, C. Grojean, D. Pappadopulo, R. Rattazzi, A. Thamm, Strong Higgs interactions at a linear collider. JHEP 02, 006 (2014). arXiv:1309.7038

58. A. S. Cornell, A. Deandrea, B. Fuks, L. Mason, Future lepton collider prospects for a ubiquitous composite pseudoscalar. Phys. Rev. D 102, 035030 (2020). arXiv:2004.09825

59. M. Chala, P. Kozów, M. Ramos , A. Titov, Effective field theory for vector-like leptons and its collider signals. Phys. Lett. B 809, 135752 (2020). arXiv:2005.09655

60. C. Degrande, C. Duhr, B. Fuks, D. Grellscheid, O. Mattelaer, T. Reiter, UFO - The Universal FeynRules Output. Comput. Phys. Commun. 183, 1201 (2012). arXiv: 1108.2040

61. N.D. Christensen, C. Duhr, FeynRules - Feynman rules made easy. Comput. Phys. Commun. 180, 1614 (2009). arXiv:0806.4194

62. A. Alloul, N.D. Christensen, C. Degrande, C. Duhr , B. Fuks, FeynRules 2.0 - A complete toolbox for treelevel phenomenology. Comput. Phys. Commun. 185, 2250 (2014). arXiv:1310.1921

63. F. Staub, SARAH 3.2: Dirac Gauginos, UFO output, and more. Comput. Phys. Commun. 184, 1792 (2013). arXiv:1207.0906

64. F. Staub, SARAH 4: A tool for (not only SUSY) model builders. Comput. Phys. Commun. 185, 1773 (2014). arXiv: 1309.7223

65. J. Alwall, R. Frederix, S. Frixione, V. Hirschi, F. Maltoni, O. Mattelaer et al., The automated computation of tree-level and next-to-leading order differential cross sections, and their matching to parton shower simulations. JHEP 07, 079 (2014). arXiv:1405.0301

66. G. Cullen et al., GOSAM - 2.0: a tool for automated one-loop calculations within the Standard Model and beyond, Eur. Phys. J. C 74, 3001 (2014). arXiv:1404.7096

67. Sherpa collaboration, Event Generation with Sherpa 2.2. SciPost Phys. 7, 034 (2019). arXiv:1905.09127

68. W. Kilian, T. Ohl, J. Reuter, WHIZARD: simulating multi-particle processes at LHC and ILC. Eur. Phys. J. C 71, 1742 (2011). arXiv:0708.4233

69. N.D. Christensen, C. Duhr, B. Fuks, J. Reuter, C. Speckner, Introducing an interface between WHIZARD and FeynRules. Eur. Phys. J. C 72, 1990 (2012). arXiv:1010.3251

70. J. Bellm et al., Herwig + 2.7 Release Note (2013). arXiv:1310.6877

71. A. Belyaev, N. D. Christensen ,A. Pukhov, CalcHEP 3.4 for collider physics within and beyond the Standard Model. Comput. Phys. Commun. 184, 1729 (2013). arXiv:1207.6082

72. M. Bondarenko, A. Belyaev, J. Blandford, L. Basso, E. Boos, V. Bunichev et al., High Energy Physics Model Database : Towards decoding of the underlying theory (within Les Houches 2011: Physics at TeV Colliders New Physics Working Group Report) (2011). arXiv:1203.1488

73. D. Barducci , L. Panizzi, Vector-like quarks coupling discrimination at the LHC and future hadron colliders. JHEP 12, 057 (2017). arXiv:1710.02325

74. X. Cid Vidal et al., Report from Working Group 3: Beyond the Standard Model physics at the HL-LHC and HE-LHC. CERN Yellow Rep. Monogr. 7, 585 (2019). arXiv:1812.07831

75. H. Zhou, N. Liu, Polarization of top quark in vector-like quark decay. Phys. Lett. B 791, 1 (2019). arXiv:1901.02300

76. B. Fuks , H.-S. Shao, QCD next-to-leading-order predictions matched to parton showers for vectorlike quark models. Eur. Phys. J. C 77, 135 (2017). arXiv:1610.04622

77. C. Degrande, Automatic evaluation of UV and R2 terms for beyond the Standard Model Lagrangians: a proof-of-principle. Comput. Phys. Commun. 197, 239 (2015). arXiv:1406.3030

78. G. Cacciapaglia, A. Carvalho, A. Deandrea, T. Flacke, B. Fuks, D. Majumder et al., Next-to-leading-order predictions for single vector-like quark production at the LHC. Phys. Lett. B 793, 206 (2019). arXiv:1811.05055

79. J. Aguilar-Saavedra, Pair production of heavy $\mathrm{Q}=$ 2/3 singlets at LHC. Phys. Lett. B 625, 234 (2005). arXiv:hep-ph/0506187

80. O. Matsedonskyi, F. Riva, T. Vantalon, Composite Charge 8/3 resonances at the LHC. JHEP 04, 059 (2014). arXiv:1401.3740

81. O. Matsedonskyi, G. Panico, A. Wulzer, On the interpretation of top partners searches. JHEP 12, 097 (2014). arXiv:1409.0100

82. A. Banfi, A. Martin, V. Sanz, Probing top-partners in Higgs+jets. JHEP 08, 053 (2014). arXiv:1308.4771

83. G. Corcella, I. Knowles, G. Marchesini, S. Moretti, K. Odagiri, P. Richardson et al., HERWIG 6: an event gen- 
erator for hadron emission reactions with interfering gluons (including supersymmetric processes). JHEP 01, 010 (2001). arXiv:hep-ph/0011363

84. G. Brooijmans et al., Les Houches 2013: Physics at TeV Colliders: New Physics Working Group Report (contribution 16) (2014). arXiv:1405.1617

85. S.R. Coleman, J. Wess, B. Zumino, Structure of phenomenological Lagrangians. 1. Phys. Rev. 177, 2239 (1969)

86. J. Callan, G. Curtis, S.R. Coleman, J. Wess, B. Zumino, Structure of phenomenological Lagrangians. 2. Phys. Rev. 177, 2247 (1969)

87. I. Brivio , M. Trott, The standard model as an effective field theory. Phys. Rept. 793, 1 (2019). arXiv:1706.08945

88. P. Artoisenet et al., A framework for Higgs characterisation. JHEP 11, 043 (2013). arXiv:1306.6464

89. I. Brivio, Y. Jiang, M. Trott, The SMEFTsim package, theory and tools. JHEP 12, 070 (2017). arXiv:1709.06492

90. C. Degrande, G. Durieux, F. Maltoni, K. Mimasu, E. Vryonidou, C. Zhang, Automated one-loop computations in the SMEFT (2020). arXiv:2008.11743

91. A. Falkowski, B. Fuks, K. Mawatari, K. Mimasu, F. Riva, V. Sanz, Rosetta: an operator basis translator for Standard Model effective field theory. Eur. Phys. J. C 75, 583 (2015). arXiv:1508.05895

92. J. Aebischer et al., WCxf: an exchange format for Wilson coefficients beyond the Standard Model. Comput. Phys. Commun. 232, 71 (2018). arXiv:1712.05298

93. B. Henning, X. Lu, H. Murayama, How to use the Standard Model effective field theory. JHEP 01, 023 (2016). arXiv: 1412.1837

94. D. Marzocca et al., BSM benchmarks for effective field theories in Higgs and electroweak physics (2020). arXiv:2009.01249

95. D. Liu, I. Low, Z. Yin, Universal Relations in Composite Higgs Models. JHEP 05, 170 (2019). arXiv:1809.09126

96. S. De Curtis, M. Redi, A. Tesi, The 4D composite Higgs. JHEP 04, 042 (2012). arXiv:1110.1613

97. D. Barducci, A. Belyaev, S. De Curtis, S. Moretti, G.M. Pruna, Exploring Drell-Yan signals from the 4D Composite Higgs Model at the LHC. JHEP 04, 152 (2013). arXiv: 1210.2927

98. D. Buarque Franzosi, G. Cacciapaglia, H. Cai, A. Deandrea, M. Frandsen, Vector and axial-vector resonances in composite models of the Higgs boson. JHEP 11, 076 (2016). arXiv:1605.01363

99. The code can be found at http://jaguilar.web.cern.ch/ jaguilar/protos/

100. The model is available in the FeynRules website at at http://feynrules.irmp.ucl.ac.be/wiki/VLQ

101. The model is available in the HEPMDB website at https://hepmdb.soton.ac.uk/hepmdb:0214.0153

102. E. Conte, B. Fuks, G. Serret, MadAnalysis 5, A Userfriendly framework for collider phenomenology. Comput. Phys. Commun. 184, 222 (2013). arXiv:1206.1599

103. E. Conte, B. Dumont, B. Fuks, C. Wymant, Designing and recasting LHC analyses with MadAnalysis 5. Eur. Phys. J. C 74. 3103 (2014). arXiv:1405.3982

104. M. Drees, H. Dreiner, D. Schmeier, J. Tattersall, J.S. Kim, CheckMATE: Confronting your favourite new physics model with LHC data. Comput. Phys. Commun. 187, 227 (2015). arXiv:1312.2591

105. D. Dercks, N. Desai, J.S. Kim, K. Rolbiecki, J. Tattersall , T. Weber, CheckMATE 2: From the model to the limit. Comput. Phys. Commun. 221, 383 (2017). arXiv:1611.09856

106. ATLAS collaboration, Search for supersymmetry at $\sqrt{s}=13 \mathrm{TeV}$ in final states with jets and two samesign leptons or three leptons with the ATLAS detector. Eur. Phys. J. C 76, 259 (2016). arXiv:1602.09058

107. Search for production of vector-like top quark pairs and of four top quarks in the lepton-plus-jets final state in $p p$ collisions at $\sqrt{s}=13 \mathrm{TeV}$ with the ATLAS detector (2016)

108. CMS collaboration, Search for pair-produced three-jet resonances in proton-proton collisions at $\sqrt{s}=13 \mathrm{TeV}$. Phys. Rev. D 99, 012010 (2019). arXiv:1810.10092

109. Y. Kang, J. Kim, J. Choi ,S. Yun, Implementation of a search for pair-produced resonances decaying each into a trijet system (36 fb-1; CMS-EXO-17-030) (2020). https://doi.org/10.14428/DVN/GAZACQ

110. CMS collaboration, Search for an exotic decay of the Higgs boson to a pair of light pseudoscalars in the final state with two muons and two b quarks in pp collisions at $13 \mathrm{TeV}$. Phys. Lett. B 795, 398 (2019). arXiv:1812.06359

111. J.-B. Lee, J. Lee, Re-implementation of a search for Higgs boson exotic decays into 2 muons and 2 b-jets (35.9 fb-1; CMS-HIG-18-011) (2020). https://doi.org/ $10.14428 / \mathrm{DVN} / \mathrm{UOH} 6 \mathrm{BF}$

112. CMS collaboration, Search for light bosons in decays of the $125 \mathrm{GeV}$ Higgs boson in proton-proton collisions at $\sqrt{s}=8 \mathrm{TeV}$. JHEP 10, 076 (2017). arXiv:1701.02032

113. CMS collaboration, Search for standard model production of four top quarks with same-sign and multilepton final states in proton-proton collisions at $\sqrt{s}=13 \mathrm{TeV}$. Eur. Phys. J. C 78, 140 (2018). arXiv:1710.10614

114. CMS collaboration, Search for production of four top quarks in final states with same-sign or multiple leptons in proton-proton collisions at $\sqrt{s}=13 \mathrm{TeV}$. Eur. Phys. J. C 80, 75 (2020). arXiv:1908.06463

115. L. Darmé, B. Fuks, MadAnalysis5 implementation of the four-top analysis of CMS with $35.9 \mathrm{fb}-1$ of data (CMS-TOP-17-009) (2021)

116. L. Darmé, B. Fuks, Re-implementation of a search for four-top quark production with leptonic final states (137 fb-1; CMS-TOP-18-003) (2020). https://doi.org/ 10.14428/DVN/OFAE1G

117. J.M. Butterworth, D. Grellscheid, M. Krämer, B. Sarrazin , D. Yallup, Constraining new physics with collider measurements of Standard Model signatures. JHEP 03, 078 (2017). arXiv:1606.05296

118. A. Buckley, J. Butterworth, L. Lonnblad, D. Grellscheid, H. Hoeth, J. Monk et al., Rivet user manual. Comput. Phys. Commun. 184, 2803 (2013). arXiv:1003.0694

119. G. Cacciapaglia, A. Deandrea, D. Harada, Y. Okada, Bounds and decays of new heavy vector-like top partners. JHEP 11, 159 (2010). arXiv:1007.2933

120. G. Cacciapaglia, A. Deandrea, L. Panizzi, N. Gaur, D. Harada, Y. Okada, Heavy Vector-like Top Partners at the LHC and flavour constraints. JHEP 03, 070 (2012). arXiv:1108.6329 
121. C.-Y. Chen, S. Dawson, E. Furlan, Vectorlike fermions and Higgs effective field theory revisited. Phys. Rev. D 96, 015006 (2017). arXiv:1703.06134

122. S. Moretti, D. O'Brien, L. Panizzi , H. Prager, Production of extra quarks at the Large Hadron Collider beyond the Narrow Width Approximation. Phys. Rev. D 96, 075035 (2017). arXiv:1603.09237

123. S. Moretti, D. O'Brien, L. Panizzi , H. Prager, Production of extra quarks decaying to Dark Matter beyond the Narrow Width Approximation at the LHC. Phys. Rev. D 96, 035033 (2017). arXiv:1705.07675

124. A. Carvalho, S. Moretti, D. O'Brien, L. Panizzi, H. Prager, Single production of vectorlike quarks with large width at the Large Hadron Collider. Phys. Rev. D 98, 015029 (2018). arXiv:1805.06402
125. CMS collaboration, Search for single production of a vector-like $\mathrm{T}$ quark decaying to a $\mathrm{Z}$ boson and a top quark in proton-proton collisions at $\sqrt{s}=13 \mathrm{TeV}$. Phys. Lett. B 781, 574 (2018). arXiv:1708.01062

126. CMS collaboration, Search for single production of vector-like quarks decaying to a b quark and a Higgs boson. JHEP 06, 031 (2018). arXiv:1802.01486

127. CMS collaboration, Search for single production of vector-like quarks decaying to a top quark and a $\mathrm{W}$ boson in proton-proton collisions at $\sqrt{s}=13 \mathrm{TeV}$. Eur. Phys. J. C 79, 90 (2019). arXiv:1809.08597

128. CMS collaboration, Search for electroweak production of a vector-like $\mathrm{T}$ quark using fully hadronic final states. JHEP 01, 036 (2020). arXiv:1909.04721 